\title{
CD2Sys: Um Framework para a Aprendizagem Experiencial de Modelagem de Sistemas com UML
}

\author{
Carlos Bahia, Bruno Gadelha
}

\author{
Instituto de Computação - Universidade Federal do Amazonas (UFAM) \\ CEP 69080-900 - Manaus - AM - Brasil \\ \{cphmb, bruno@i comp.ufam.edu.br
}

\begin{abstract}
This paper presents an investigation about the use of Experiential Learning Theory (APT) in a system modeling course with UML. The investigation was performed according to the Design Science Research (DSR) approach that presupposes the generation of knowledge through the development of artifacts. Thus, the artifact developed was CD2Sys, a framework for generating Web systems from UML classes diagrams. An experimental study was conducted in a Systems Analysis and Design course in the Federal University of Amazonas. As a result, in addition to the developed artifact, there is the acceptance of the developed technology as an instrument for experiencing software models in real time in the classroom.
\end{abstract}

Resumo. Este artigo apresenta uma investigação através do Design Science Research (DSR) acerca do uso da Teoria da Aprendizagem Experiencial (TAE) em um curso de modelagem de sistemas com UML. A DSR pressupõe a geração de conhecimento através do desenvolvimento de artefatos. Assim, o artefato desenvolvido foi o CD2Sys, um framework de geração de sistemas Web a partir de diagramas de classes UML. Foi desenvolvido um estudo experimental em uma turma de Análise e Projeto de Sistemas na Universidade Federal do Amazonas. Como resultados, além do artefato desenvolvido, temse a aceitação da tecnologia desenvolvida como instrumento de experimentação de modelos em tempo real em sala de aula.

\section{Introdução}

Modelar sistemas é complexo/complicado (Gogolla e Stevens, 2018; Laroza e Seabra, 2015) dado que consiste em uma atividade que requer habilidades de abstração que são essenciais para o exercício da profissão dos engenheiros de software. Porém, desenvolver tais habilidades apresenta-se um desafio para educadores (Engels et. al., 2005). No contexto dos cursos de modelagem de sistemas, geralmente os conceitos, modelos, seus elementos e sintaxe são apresentados aos alunos em aulas tradicionais, e exercícios com cenários fictícios (toy problems) são resolvidos. Modelar passa a ser uma atividade de transcrição dos cenários descritos em linguagem natural para uma linguagem semiestruturada e mais formal que é a UML.

A UML (Unified Modeling Language) é uma linguagem de propósito geral para modelar software sob o paradigma da orientação a objetos. A UML não é uma linguagem de programação. Através de seus diagramas, a UML fornece múltiplas visões do sistema modelado, onde cada diagrama apresenta um aspecto complementar ao outro (Guedes, 2011). Um dos diagramas da UML mais utilizados é o diagrama de classes. Ben Abdessalem Karaa et. al. (2016) destacam que os diagramas de classes aumentam o conhecimento sobre o sistema modelado durante todo o processo de desenvolvimento de software, servindo de ponte entre as informações de domínio do negócio, do lado do usuário, e os componentes de software, do lado do engenheiro de software. 
VII Congresso Brasileiro de Informática na Educação (CBIE 2018)

Anais do XXIX Simpósio Brasileiro de Informática na Educação (SBIE 2018)

Assim, considerando as dificuldades no processo de ensino/aprendizagem de modelagem de sistemas e a importância do desenvolvimento dessas habilidades para o exercício profissional de futuros engenheiros de software, este trabalho apresenta uma pesquisa acerca do uso, em sala de aula, de um framework de geração de sistemas a partir de modelos de diagramas de classes UML. O framework proposto, chamado CD2Sys, serve como plataforma de experimentação de modelos no contexto da aprendizagem experiencial em cursos de Análise e Projeto de Sistemas.

Este artigo está organizado da seguinte forma: a Seção 2 consiste na fundamentação teórica da pesquisa apresentando os conceitos da Teoria da Aprendizagem Experiencial e descrevendo alguns trabalhos relacionados a esta; a Seção 3 apresenta a metodologia utilizada na pesquisa; a Seção 4 apresenta framework CD2Sys desenvolvido; a Seção 5 apresenta como o CD2Sys foi utilizado e avaliado em sala de aula e, por fim, a Seção 6 apresenta as considerações finais.

\section{Fundamentação Teórica}

Esta seção apresenta a Teoria da Aprendizagem Experiencial na qual esta pesquisa está baseada. Na sequência são apresentados alguns trabalhos relacionados.

\subsection{Teoria da Aprendizagem Experiencial}

A teoria da aprendizagem experiencial (TAE) consiste em uma visão dinâmica de aprendizagem baseada em um ciclo de aprendizado impulsionado pela resolução da dupla dialética ação/reflexão e experiência/abstração. A teoria baseia-se no trabalho de estudiosos proeminentes do século XX que deram à experiência um papel central em suas teorias da aprendizagem e desenvolvimento humanos - notavelmente John Dewey, Kurt Lewin, Jean Piaget, William James, Carl Jung, Paulo Freire, Carl Rogers e outros para desenvolver uma dinâmica, modelo holístico do processo de aprendizagem da experiência e um modelo multilinear de desenvolvimento de indivíduos adultos. A TAE define a aprendizagem como o principal processo de adaptação humana envolvendo a pessoa como um todo. Dessa forma, a TAE é aplicável não só na educação formal sala de aula, mas em todas as áreas da vida. O processo de aprender com a experiência é onipresente, presente na atividade humana em todos os lugares e a toda hora (Kolb e Kolb, 2009).

A TAE baseia-se em seis proposições, a saber:

- Aprender é melhor concebido como um processo, não em termos de resultados. Deve-se focar em um processo que melhor garanta a sua aprendizagem e levar em consideração o esforço e eficácia de sua aprendizagem.

- Todo aprendizado é reaprendizado. A aprendizagem é melhor facilitada por um processo que extrai as crenças e ideias dos alunos sobre um tópico, para que possam ser examinados, testados e integrados com ideias novas e mais refinadas.

- Aprendizagem requer a resolução de conflitos entre modos de adaptação dialeticamente opostos para o mundo. Conflito, diferenças e discordância impulsionam o processo de aprendizagem. Indivíduos devem lidar com diferenças de pensamento, sentimentos e atitudes.

- A aprendizagem é um processo holístico de adaptação. Não é apenas o resultado da cognição, mas envolve o funcionamento integrado do indivíduo pensar, sentir, perceber e comportar-se. Abrange outras especialidades em meio a modelos de adaptação do método científico à resolução de problemas, tomada de decisão e criatividade.

- Resultados de aprendizagem de transações sinérgicas entre a pessoa e o meio ambiente. A aprendizagem tende a ser mais duradoura quando há uma 
VII Congresso Brasileiro de Informática na Educação (CBIE 2018)

Anais do XXIX Simpósio Brasileiro de Informática na Educação (SBIE 2018)

maior interação entre o indivíduo e seu ambiente. A forma que cada um processa as experiências vividas determina as opções e decisões a serem tomadas. Assim, a aprendizagem acontece a partir das escolhas vividas.

- Aprendizagem é o processo de criação de conhecimento. TAE propõe uma teoria construtivista de aprendizagem onde o conhecimento social é criado e recriado no conhecimento pessoal do aluno.

\subsection{Trabalhos Relacionados}

Uma plataforma de experimentação de modelos UML para ser usado em cursos de modelagem de sistemas em uma perspectiva da aprendizagem experiencial requer a geração de sistemas a partir dos modelos desenvolvidos. Isso vai em direção das pesquisas na área do desenvolvimento dirigido a modelos (MDD - Model Drivel Development). O MDD busca diminuir as distâncias entre os modelos desenvolvidos e os software implementados (Van den Brand e Groote, 2012). Assim, os trabalhos a seguir são resultados de pesquisas em MDD que consideram a geração de sistemas de software a partir de modelos descritos com UML.

Garzón et. al. (2015) apresenta o Umple, que consiste em um framework para o desenvolvimento orientado a modelos de sistemas orientados a objetos. O Umple possibilita a geração de sistemas a partir de modelos descritos textualmente de acordo com sua própria linguagem. Ele oferece também a possibilidade de se realizar engenharia reversa, onde modelos são gerados a partir do código-fonte dos sistemas orientados a objetos. Este trabalho diferencia-se da pesquisa apresentada neste artigo principalmente pelo fato dele trabalhar com uma linguagem textual própria para a representar os modelos. Adotá-lo como ferramenta de suporte à aprendizagem experiencial requer a aprendizagem desta linguagem específica que não é padrão nem na academia e nem na indústria de desenvolvimento.

Mais especificamente com relação a diagramas de classes descritos com UML, Sadaf et. al. (2016) apresentam o FED-CASE que consiste em uma ferramenta pra convertê-los em código estrutural. Esse código estrutural não é o código-fonte da aplicação em uma linguagem de programação. Ele consiste em uma linguagem intermediária que deve ser interpretada a-posteriori e convertida em linguagem de programação. Assim, a ferramenta em questão não gera um sistema utilizável a partir do diagrama de classes modelado.

No contexto do desenvolvimento para dispositivos móveis, Parada et. al. (2015) propõem a automatização do desenvolvimento de aplicações móveis com Android e Windows Phone a partir de diagramas de classes e de sequência da UML. A abordagem é similar à proposta neste artigo no que tange à geração de software executável a partir de modelos. Porém, a plataforma desenvolvida pelos autores é de difícil adoção para uso com alunos iniciantes em modelagem de sistemas, visto que os diagramas de entrada da plataforma (classes e sequência) devem refletir às estruturas internas de implementação de aplicativos móveis e não apenas classes de domínio. Esta poderia ser utilizada como plataforma de experimentação em cursos mais avançados de desenvolvimento móvel onde os alunos conhecem as particularidades desse tipo de desenvolvimento e já possuem domínio da notação avançada da UML.

\section{Metodologia}

A metodologia utilizada na pesquisa é a Design Science Research (DSR). De acordo com Dresch et. al. (2015), a DSR consiste em uma forma de produção de conhecimento científico que envolve o desenvolvimento de uma inovação, com a intenção resolver problemas do mundo real e, ao mesmo tempo, fazer uma contribuição científica de caráter prescritivo. De forma bem resumida, a DSR prevê as seguintes atividades: 
VII Congresso Brasileiro de Informática na Educação (CBIE 2018)

Anais do XXIX Simpósio Brasileiro de Informática na Educação (SBIE 2018)

definição do problema a ser resolvido; proposta de artefato para solução do problema; desenvolvimento e avaliação do artefato; conclusões e divulgação dos resultados.

O problema considerado na pesquisa consiste na dificuldade da aprendizagem de modelagem de sistemas, uma vez que esta requer habilidades de abstração para representação de elementos do mundo real em linguagens semiestruturadas. Essas habilidades são essenciais para futuros engenheiros de software que devem identificar problemas a serem resolvidos computacionalmente e desenvolver tais soluções através do desenvolvimento de sistemas onde os conceitos relevantes dos problemas devem estar formalmente descritos e representados. Desenvolver a habilidade de abstração é um desafio para professores de cursos de computação.

$\mathrm{O}$ artefato proposto para estudar o problema levantado consiste no CD2Sys, um framework de geração de sistemas para a plataforma Web a partir de descrições dos conceitos mais relevantes do domínio representados em diagramas de classes descritos em UML. O artefato está descrito em detalhes na Seção 4. O objetivo do framework é de servir de plataforma de experimentação de modelos, diminuindo os gaps de abstração dos conceitos do domínio modelado e o sistema concreto derivado. O CD2Sys foi desenvolvido para a plataforma Web e gera sistemas para a mesma plataforma através do framework de desenvolvimento Yii2, um dos mais utilizados pela indústria de desenvolvimento de software.

Para avaliar o artefato proposto, realizou-se um estudo experimental com uma turma de Análise e Projeto de Sistemas do curso de Engenharia de Software da Universidade Federal do Amazonas. O estudo envolveu 15 alunos matriculados no curso que tiveram que, a partir de uma descrição de um domínio, desenvolver um diagrama de classes usando UML e fazer a geração do sistema modelado utilizando o CD2Sys. Ao final da atividade, os alunos responderam a um questionário segundo o Modelo de Aceitação de Tecnologia (TAM - Technology Acceptance Model) (Venkatesh e Bala, 2008) para verificar a utilidade, a facilidade e a intenção de uso da tecnologia desenvolvida. Além disso, questões abertas sobre a experiência de adoção do CD2Sys em sala de aula foram feitas aos alunos. Por fim, os pesquisadores conversaram com cada um dos alunos para que tivessem a oportunidade de dar opiniões que não foram contempladas no questionário. As questões abertas e as entrevistas foram analisadas segundo o MEDS - Método da Explicitação do Discurso Subjacente (Nicolaci-da-costa, 2007). Essa avaliação é descrita em detalhes na Seção 5.

\section{CD2Sys: Do Diagrama de Classes ao Sistema Implementado}

Uma questão recorrente apresentada pelos alunos diz respeito à dificuldade de visualização do que será derivado a partir da modelagem realizada. Muitos dos alunos não conseguem ver diretamente que os modelos devem ser refletidos na implementação do sistema. Isso motivou a concepção do CD2Sys que consiste em um framework de geração de sistemas a partir de diagramas de classes UML. O objetivo do CD2Sys é possibilitar ao aluno perceber os efeitos de sua modelagem na implementação de um sistema utilizável. Com isso, o aluno pode refletir sobre sua modelagem, refiná-la ou corrigi-la, e verificar o resultado de suas intervenções no modelo diretamente no sistema final. Executar o sistema modelado possibilita ao aluno verificar se sua modelagem atende aos requisitos descritos nos cenários propostos pelo professor.

A Figura 1 ilustra o processo de geração de um sistema a partir de um diagrama de classes UML. Uma vez desenvolvido o diagrama de classes, o aluno precisa exportar seu diagrama para o formato XML através da ferramenta que usou para elaborar seu diagrama. De posse do arquivo XML, o aluno deve acessar o CD2Sys e importar o arquivo XML para o sistema de geração. O CD2Sys, por sua vez, internamente analisa o arquivo enviado, faz as transformações do modelo de classes a um modelo relacional, conforme Blaha e Rumbaugh (2006), para criação do banco de dados da aplicação. Uma vez criado o banco de dados, o CD2Sys utiliza as ferramentas do Yii2 Framework para 
VII Congresso Brasileiro de Informática na Educação (CBIE 2018)

Anais do XXIX Simpósio Brasileiro de Informática na Educação (SBIE 2018)

a geração das funcionalidades de cadastro, alteração, exclusão e consulta das tabelas geradas. Uma vez gerado, o sistema é apresentado para avaliação do aluno.

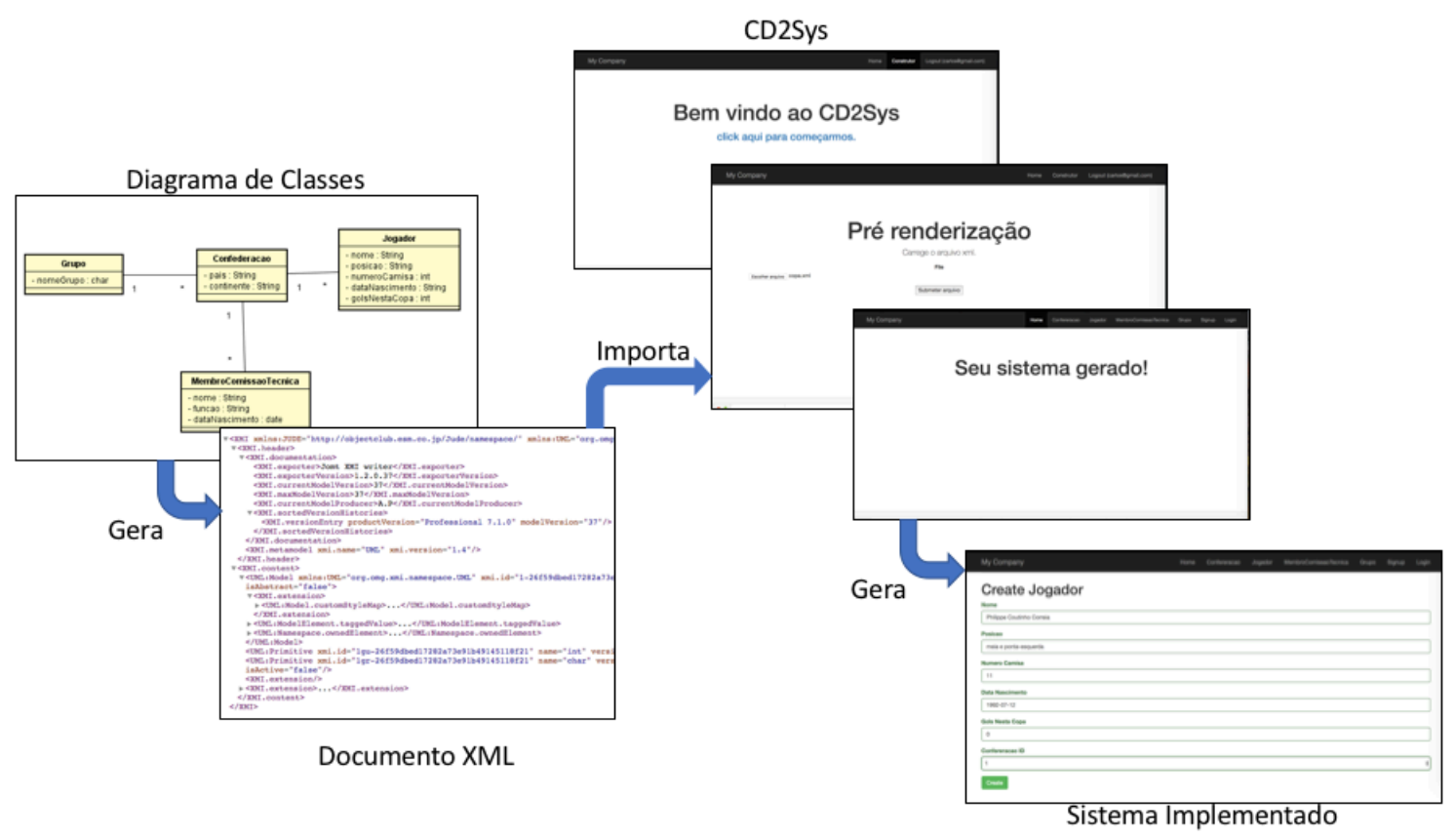

Figura 1. Processo de geração de um sistema com o CD2Sys

Uma vez gerado, o sistema pode ser utilizado pelo aluno. Ao utilizar o sistema, o aluno observa se o mesmo atende aos requisitos do cenário considerado e se reflete a modelagem desenvolvida. Assim, o aluno consegue verificar, inclusive, problemas na modelagem que foram evidenciadas no uso do sistema, fazendo-o refletir sobre a modelagem realizada, validá-la, avalia-la e refazê-la até que o sistema derivado esteja de acordo com o desejado. Dessa forma, o CD2Sys serve como uma plataforma de experimentação de modelos de classes, possibilitando a visualização concreta do sistema modelado.

\section{Avaliação do CD2Sys}

Um estudo experimental com 15 alunos da disciplina Análise de Projeto de Sistemas do curso de Engenharia de Software da Universidade Federal do Amazonas. O estudo teve como objetivo verificar se a adoção de uma metodologia experiencial em sala de aula impactaria positivamente na aprendizagem de diagramas de classes da UML e no desenvolvimento da habilidade de abstração dos alunos.

Como preparação para o estudo, solicitou-se aos alunos a instalação e configuração do CD2Sys e da ferramenta Astah em seus computadores. A ferramenta Astah foi utilizada para o desenvolvimento do diagrama de classes e sua exportação para XML. Além disso, foi elaborado um cenário de um sistema de gestão de séries de TV para que os alunos fizessem a modelagem.

Uma vez instalados os sistemas nos computadores dos alunos, estes foram divididos em 6 duplas e 1 trio. A divisão foi feita de maneira espontânea entre os próprios alunos. Então, foi dada uma cópia do cenário elaborado a cada um dos alunos que tinham que: ler, compreender, extrair os conceitos mais relevantes e elaborar um diagrama de classes UML utilizando o Astah como ferramenta de modelagem. Para tanto, foi reservado um tempo de 40 minutos. Nesse tempo, eles poderiam interagir com sua dupla ou trio da forma que achasse melhor para fazer o seu modelo. Terminado o tempo, foi solicitado que cada dupla exportasse o seu modelo para um arquivo.XML e 
VII Congresso Brasileiro de Informática na Educação (CBIE 2018)

Anais do XXIX Simpósio Brasileiro de Informática na Educação (SBIE 2018)

o importasse no CD2Sys. Então, cada dupla deveria usar o sistema gerado pelo CD2Sys e verificar se o sistema correspondia à modelagem que haviam feito, observado aspectos como: nome de classes, tipos de dados, relação entre as classes e cardinalidade entre elas. Se o sistema gerado estivesse dentro das especificações do cenário, a dupla ou trio poderia dar por encerrada a atividade. Caso contrário, eles deveriam refletir e alterar o modelo desenvolvido e refazer a geração do sistema. Isso deveria acontecer até que o sistema satisfizesse todos os requisitos do cenário.

Ao final os alunos foram submetidos a um questionário segundo o TAM (Venkatesh e Bala, 2008) com questões fechadas em escala Likert de 7 pontos relacionadas à utilidade percebida, facilidade e intenção de uso do CD2Sys. Além disso, deveriam responder a questões abertas sobre sua experiência no uso do CD2Sys e sua aplicabilidade nas aulas de modelagem. A Tabela 1 apresenta as questões respondidas pelos alunos ao final do estudo.

Tabela 1. Questionário respondido pelos alunos ao fim do estudo.

\begin{tabular}{|c|c|c|}
\hline \multicolumn{2}{|r|}{ Utilidade Percebida } & Tipo \\
\hline UP1 & Usar o CD2Sys melhora o meu entendimento sobre o diagrama de classes. & Likert de 7 pontos \\
\hline UP2 & $\begin{array}{l}\text { Usar o CD2Sys melhora a minha capacidade de abstração dos conceitos do domínio do } \\
\text { sistema. }\end{array}$ & Likert de 7 pontos \\
\hline UP3 & $\begin{array}{l}\text { Usar o CD2Sys me permite avaliar completamente a aderência do modelo que desenvolvi ao } \\
\text { sistema gerado. }\end{array}$ & Likert de 7 pontos \\
\hline UP4 & $\begin{array}{l}\text { Eu acho o CD2Sys útil como ferramenta de experimentação para dar suporte ao meu } \\
\text { aprendizado de diagrama de classes. }\end{array}$ & Likert de 7 pontos \\
\hline \multicolumn{3}{|c|}{ Facilidade de Uso Percebida } \\
\hline FUP1 & $\mathrm{O}$ CD2Sys foi claro e fácil de entender. & Likert de 7 pontos \\
\hline FUP2 & Usar o CD2Sys não demandou muito esforço mental. & Likert de 7 pontos \\
\hline FUP3 & Eu acho o Cd2Sys fácil de usar. & Likert de 7 pontos \\
\hline FUP4 & Eu acho fácil gerar sistemas baseados em diagramas de classes com o CD2Sys. & Likert de 7 pontos \\
\hline \multicolumn{3}{|c|}{ Intenção de Uso } \\
\hline IU1 & $\begin{array}{l}\text { Assumindo que tenha acesso ao CD2Sys, eu pretendo usá-lo para verificar como seria o } \\
\text { sistema modelado pelo meu diagrama de classes. }\end{array}$ & Likert de 7 pontos \\
\hline IU2 & $\begin{array}{l}\text { Dado que eu tenha acesso ao CD2Sys, eu prevejo que eu a usaria para verificar a corretude } \\
\text { dos meus diagramas de classes. }\end{array}$ & Likert de 7 pontos \\
\hline IU3 & $\begin{array}{l}\text { Eu pretendo usar o CD2Sys como ferramenta de prototipação rápida visando consolidar meu } \\
\text { aprendizado de modelagem de sistemas. }\end{array}$ & Likert de 7 pontos \\
\hline \multicolumn{3}{|c|}{ Experiência Geral } \\
\hline EX1 & $\begin{array}{l}\text { Você acha que a ferramenta utilizada ajuda a abstrair ou entender os conceitos relevantes do } \\
\text { domínio explorado? Comente. }\end{array}$ & Questão aberta \\
\hline EX2 & $\begin{array}{l}\text { Você acha que poder visualizar um sistema logo após efetuar sua modelagem ajuda no seu } \\
\text { entendimento sobre o modelo? Comente. }\end{array}$ & Questão aberta \\
\hline EX3 & $\begin{array}{l}\text { Ao ver o sistema resultante de sua modelagem, você precisou refazer o seu modelo? Por } \\
\text { que? }\end{array}$ & Questão aberta \\
\hline EX4 & $\begin{array}{l}\text { Comente os pontos positivos e negativos de ter uma ferramenta de geração de sistemas nas } \\
\text { aulas de modelagem. }\end{array}$ & Questão aberta \\
\hline
\end{tabular}

\subsection{Análise dos Dados}

Os dados dos questionários respondidos pelos alunos foram consolidados e estão apresentados na Tabela 2. Os números correspondem à quantidade de alunos que escolheram uma opção na escala Likert de 7 pontos.

\section{Tabela 2. Dados do questionário consolidados.}

\begin{tabular}{|c|c|c|c|c|c|c|c|}
\hline Utilidade Percebida & 1 & 2 & 3 & 4 & 5 & 6 & 7 \\
\hline O uso do CD2Sys melhora o seu entendimento sobre diagrama de classes & & & & 3 & 6 & 4 & 2 \\
\hline $\begin{array}{l}\text { Usar o CD2Sys melhorou a sua capacidade de abstrair os conceitos do domínio } \\
\text { do sistema }\end{array}$ & & & & 4 & 4 & 5 & 1 \\
\hline $\begin{array}{l}\text { Usar o CD2Sys o permitiu completamente a aderência do modelo que } \\
\text { desenvolveu ao sistema gerado }\end{array}$ & & 1 & & 3 & 3 & 5 & 3 \\
\hline $\begin{array}{l}\text { Achou que CD2Sys útil como ferramenta de experimentação para suporte ao } \\
\text { aprendizado de diagrama de classes }\end{array}$ & & & 1 & & 4 & 5 & 5 \\
\hline \multicolumn{8}{|l|}{ Facilidade de uso } \\
\hline O CD2Sys foi claro e fácil de entender & 1 & 2 & 2 & 3 & 5 & 2 & \\
\hline Usar o CD2Sys não demandou muito esforço mental & 1 & 2 & 1 & 2 & 3 & 6 & 1 \\
\hline Achou fácil de usar & 1 & 2 & 3 & 2 & 3 & 2 & 2 \\
\hline Achou fácil de gerar sistemas baseados em diagramas com o CD2Sys & 1 & 2 & 2 & 1 & 4 & 3 & 2 \\
\hline
\end{tabular}


VII Congresso Brasileiro de Informática na Educação (CBIE 2018)

Anais do XXIX Simpósio Brasileiro de Informática na Educação (SBIE 2018)

\begin{tabular}{|c|c|c|c|c|c|}
\hline Intenção de uso & & & & & \\
\hline $\begin{array}{l}\text { Assumindo que você tem acesso ao CD2Sys, você pretende usá-lo para verificar } \\
\text { como seria o sistema modelado de um diagrama de classes }\end{array}$ & 1 & 2 & 1 & 7 & 4 \\
\hline $\begin{array}{l}\text { Dado que tem acesso ao sistema, pretende usar para verificar a corretude dos } \\
\text { seus modelos de diagrama de classes }\end{array}$ & & 2 & 1 & 5 & 7 \\
\hline $\begin{array}{l}\text { Pretende usar o CD@sys como ferramenta de prototipação rápida visando } \\
\text { consolidar o aprendizado de modelagem de sistemas }\end{array}$ & & 3 & 4 & 5 & 4 \\
\hline
\end{tabular}

Analisando os dados, verifica-se que os alunos perceberam a utilidade do CD2Sys como framework de experimentação de modelos UML, destacando sua utilidade na melhoria da capacidade de abstração dos alunos. Os alunos conseguiram verificar a aderência do sistema implementado ao modelo por eles desenvolvidos. Em geral, o CD2Sys pode melhorar seu entendimento sobre o diagrama de classes. Com relação à facilidade de uso do $\mathrm{CD} 2$ Sys, verifica-se que não há uma concordância entre os alunos neste quesito. Os alunos acharam o framework difícil de usar. Porém, verificase que, apesar da dificuldade percebida, os alunos o utilizariam como plataforma de experimentação de diagramas de classes. A intenção de uso ficou mais evidente no quesito de verificação da corretude do modelo, indicando que os alunos gostaram da possibilidade de ver o efeito dos seus erros de modelagem no sistema implementado. Isso dá a eles a segurança para refinar o modelo, e como consequência, aumenta sua aprendizagem sobre modelagem de sistemas.

As questões abertas foram analisadas de acordo com o MEDS (Nicolaci-daCosta, 2007). A Tabela 3 apresenta as categorias que emergiram dos dados analisados bem como a quantidade de citações que as originaram. As categorias são comentadas a seguir.

Tabela 3. Categorias que emergiram das questões abertas do questionário.

\begin{tabular}{|c|l|c|}
\hline$\#$ & \multicolumn{1}{|c|}{ Categoria } & Citações \\
\hline 1 & Ferramenta útil para entender elementos do domínio & 9 \\
\hline 2 & Visualizar sistema ajuda a entender o modelo & 5 \\
\hline 3 & Possibilidade de refinar o modelo & 4 \\
\hline 4 & Feedback rápido sobre o modelo & 4 \\
\hline
\end{tabular}

A categoria 1 diz respeito à utilidade da ferramenta no suporte ao entendimento dos elementos do domínio a ser modelado. Esta categoria está relacionada à capacidade de abstração e possibilidade de visualização prática dos elementos modelados pelos alunos. Afirmativas como "Foi possível visualizar na prática e como o diagrama influencia no sistema" e "Ajudou a ter uma visão mais concreta do diagrama implementado" corroboram com essa ideia.

A categoria 2 também está relacionada à capacidade de abstração dos alunos. Ela evidencia a necessidade de os alunos verem na prática o sistema implementado a partir do modelo que desenvolveram. Os alunos afirmam que verificar que o sistema gerado segue fielmente a modelagem desenvolvida os ajudam a entender os erros cometidos durante a modelagem. Afirmativas como "o resultado foi igual o da modelagem" e "mostrou ser fiel ao que foi feito no diagrama" exemplificam o fato do sistema espelhar o modelo. As afirmações "mostrou os erros logo em seguida da diagramação" e "ajudou a encontrar falhas no modelo" exemplificam a necessidade de experimentação do modelo e a importância disso na construção do entendimento sobre a modelagem.

A categoria 3, sobre a possibilidade de refinar o modelo gerado, surge como uma resposta à questão que norteou o estudo: a viabilidade de uma abordagem experiencial no ensino de modelagem de sistemas. Os alunos destacaram a importância 
VII Congresso Brasileiro de Informática na Educação (CBIE 2018)

Anais do XXIX Simpósio Brasileiro de Informática na Educação (SBIE 2018)

de experimentar o modelo e, a partir do feedback fornecido pelo sistema gerado, identificar os erros de modelagem cometidos, reagir e refazer o modelo. Algumas afirmações que reforçam nossa análise: "refiz o modelo porque eu vi que tinha errado o tipo dos atributos" e "errei em algumas cardinalidades". Os problemas só foram percebidos após a utilização do sistema resultante do modelo.

Por fim, a categoria 4 ressalta a importância de se ter um feedback imediato sobre a modelagem realizada. O CD2Sys, ao gerar um sistema em tempo real a partir do modelo, serve como uma resposta aos alunos sobre a corretude de seus modelos. Sobre isso, um aluno afirmou: "Dinâmico e possibilita vê logo o resultado". Outro corroborou com a ideia com a seguinte fala: "Permite que visualize logo onde houve o erro e corrija."

\section{Conclusão}

Este artigo apresentou o CD2Sys, um framework para a aprendizagem experiencial de modelagem de sistemas com UML. A Teoria Aprendizagem Experiencial (TAE) foi utilizada na disciplina de Análise e Projeto de Sistemas do curso de Engenharia de Software da Universidade Federal do Amazonas dado que propõe uma aprendizagem baseada na troca de experiências entre os alunos e as práticas vividas com o objeto de estudo. Assim, o CD2Sys foi desenvolvido de modo a servir como plataforma de experimentação de modelos UML, mais especificamente diagramas de classes.

$\mathrm{O}$ CD2Sys se apresentou como uma alternativa para dar suporte à TAE em disciplinas de modelagem de sistemas uma vez que: o aprendizado do modelo em questão (diagrama de classes) se deu a partir das várias interações entre os alunos nas tarefas de conceber o modelo, gerar o sistema utilizável, testar o sistema gerado e refinar o modelo; o aprendizado é sempre um reaprendizado a partir da identificação de problemas nos modelos gerados e a consequente reflexão para ajustes no mesmo; tais problemas identificados durante a execução do sistema gerado se apresentam como um conflito com as crenças iniciais dos alunos acerca dos modelos, o que os leva a solucioná-los; a cada iteração modelagem-geração do sistema-refino do modelo, os modelos mentais dos alunos são adaptados para acomodar o conhecimento gerado; o CD2Sys apresenta-se como uma plataforma que possibilita a interação do aluno com o objeto de estudo, resultando em uma aprendizagem mais significativa; e, por fim, a aprendizagem é o processo de criação do conhecimento, onde este acontece das interações entre os próprios alunos e entre eles e o objeto de estudo. Os aspectos citados vão ao encontro das proposições norteadoras da TAE.

Como resultado do estudo experimental do CD2Sys, tem-se a aceitação da tecnologia proposta que foi percebida pelos alunos como útil e, apesar das dificuldades enfrentadas, os alunos declararam sua intenção de uso como plataforma experimental de diagramas de classes. Com relação à metodologia baseada na aprendizagem experiencial, ficou claro que oferecer aos alunos a oportunidade de experimentar os diagramas de forma menos abstrata, através da geração do sistema modelado, possibilitou aos alunos diminuir o gap dos conceitos abstratos dos modelos e os conceitos concretos do sistema implementado. 
VII Congresso Brasileiro de Informática na Educação (CBIE 2018)

Anais do XXIX Simpósio Brasileiro de Informática na Educação (SBIE 2018)

\section{Referências}

Ben Abdessalem Karaa, W., Ben Azzouz, Z., Singh, A., Dey, N., S. Ashour, A., \& Ben Ghazala, H. (2016). Automatic builder of class diagram (ABCD): an application of UML generation from functional requirements. Software: Practice and Experience, 46(11), 1443-1458.

Blaha, M., Rumbaugh, J. (2006) Modelagem e Projetos Baseados em Objetos com UML 2. Ed. 2. Editora Elsevier. Rio de Janeiro, p 362-394.

Dresch, A.; Lacerda, D. P.; Antunes Jr, J. A. V (2015). Design Science Research: Método de Pesquisa para Avanço da Ciência e Tecnologia. Bookman.

Engels, G., Hausmann, J. H., Lohmann, M., \& Sauer, S. (2005). Teaching UML is teaching software engineering is teaching abstraction. In International Conference on Model Driven Engineering Languages and Systems (pp. 306-319). Springer, Berlin, Heidelberg.

Garzón, M. A., Aljamaan, H., \& Lethbridge, T. C. (2015). Umple: A framework for model driven development of object-oriented systems. In Software Analysis, Evolution and Reengineering (SANER), 2015 IEEE 22nd International Conference on (pp. 494-498). IEEE.

GUEDES, G. T. A. (2011) UML 2: Uma abordagem prática. 2a edição. Novatec Editora.

Gogolla, M., Stevens, P. (2018). Teaching modeling in computer science as an ecosystem: a provocative analogy. Computer Science Education, 1-18.

Kolb, A. Y., Kolb, D. A. (2009) Experiential Learning Theory: A Dynamic, Holistic Approach to Management Learning, Education and Development, In: The SAGE Handbook of Management Learning, Education and Development. Editador por: Armstrong, S.J. e Fukami, C.V. Londres: Sage, p 42-68.

Laroza, J., \& Seabra, R. (2015, October). Rea-uml: Recurso educacional aberto para ensino da uml. In Brazilian Symposium on Computers in Education (Simpósio Brasileiro de Informática na Educação-SBIE) (Vol. 26, No. 1, p. 11).

Nicolaci-da-Costa, A. M. (2007) O Campo da Pesquisa Qualitativa e o Método da Explicitação do Discurso Subjacente (MEDS). In: Psicologia: Reflexão e Crítica. vol.20 no.1. ISSN: 0102-7972. RS, Porto Alegre.

Parada, A., Marques, M., \& de Brisolara, L. B. (2015). Automating mobile application development: UML-based code generation for Android and Windows Phone. Revista de Informática Teórica e Aplicada, 22(2), p. 31-50.

Sadaf, S., Athar, A., \& Azam, F. (2016). Evaluation of FED-CASE-A Tool to Convert Class Diagram into Structural Coding. In Computer, Consumer and Control (IS3C), 2016 International Symposium on (pp. 184-187). IEEE.

Van den Brand, M. G., \& Groote, J. F. (2012). Advances in model driven software engineering. ERCIM News, 91, 23-24.

Venkatesh, V., Bala, H. (2008). Technology Acceptance Model 3 and a Research Agenda on Interventions. In: Decision Sciences, v. 38, n. 2 p 273-315. 